\title{
The Effect of Roughness on the Polarization of Thermal Emission From a Surface
}

\author{
T. Hagfors and J. Moriello \\ Lincoln Laboratory, ${ }^{1}$ Massachusetts Institute of Technology, Cambridge, Mass.
}

The observation of the polarization of the thermal emission from a largely dielectric smooth surface may be used to infer the dielectric constant of the medium. The method was suggested by Troitsky [Astron. Zhur. 31, 511, 1954] for lunar observations, and has since then actually been used to evaluate the dielectric constant of the moon by Soboleva [Astron. Zhur. 39, 1124, 1962], Heiles and Drake [Icarus 2, 281, 1963], Baars et al., [Astron. J. 70, 132, 1965], and Davies and Gardner (this meeting). The same method basically was also used by Clark and Kuzmin [private communication] to study the surface material of Venus. Quite generally the radiometrically determined dielectric constants have been consistently lower than values derived from radar data by studying cross sections [Evans and Pettengill, J. Geophys. Res. 68, 423, 1963] and Evans, private communication [1965].

In view of this persistent discrepancy, and because of many suggestions that surface roughness might play some part in the apparent disagreement, a fairly rigorous theory was developed for the emission of electromagnetic waves from a dielectric medium with an undulating boundary surface. In the following few paragraphs we briefly outline the basic procedure, state the several simplifying assumptions used, and finally present some numerical results in the form of diagrams in order to show the effect of surface roughness on the emission.

The boundary between the dielectric material and free space is plane in the mean, but deviates from this plane by an amount $Z(x, y)$ which depends on the position $(x, y)$ on the mean plane. The random variable is taken to be Gaussian with zero mean and with root-mean-square value $h_{0}$. The covariance of $Z$ is defined by

$$
\langle Z(x, y) Z(x+\Delta x, y+\Delta y)\rangle_{\mathrm{avg}}=h_{0}^{2} \rho(\Delta r)
$$

where

$$
\Delta r=\left\{\Delta x^{2}+\Delta y^{2}\right\}^{1 / 2} .
$$

The boundary conditions relating the fields of a wave inside the dielectric medium to the boundary fields immediately outside the medium are obtained by means of the Fresnel transmission coefficients

'Operated with support from the U.S. Air Force. evaluated with the local parameters (locally flat approximation). This approximation requires that the radius of curvature of the undulations be larger than the wavelength.

With the boundary conditions fixed, the fields due to a single plane wave inside the medium are evaluated for an arbitrary point of observation outside the medium by means of the Stratton-Chu formula [Stratton, Electromagnetic Theory, McGraw-Hill, 1941]. When the wave vector of the wave arriving at the observer is $\mathbf{k}$ and the wave vector of the wave inside the medium is $\kappa$, it turns out that the dominant contribution to the observed power at the observer comes from surface elements with a normal given by

$$
\mathbf{n}=\frac{\boldsymbol{\kappa}-\mathbf{k}}{|\boldsymbol{\kappa}-\mathbf{k}|}
$$

This contribution becomes relatively more important as the wavelength of the radiation is made small compared with the radius of curvature of the surface [Hagfors, J. Geophys. Res. 69, 3779, 1964].

Under the further assumption that

$$
h_{0}\left(\mathbf{e}_{z} \cdot(\boldsymbol{\kappa}-\mathbf{k}) \gg 1,\right.
$$

which corresponds to "deep" phase variations across the boundary, the integration was carried out numerically over an omnidirectional angular power spectrum (i.e., over $\boldsymbol{\kappa}$ ) of unpolarized radiation to give the amount of power polarized in the plane of incidence $\left(P_{\|}\right)$and across the plane of incidence $\left(P_{\perp}\right)$ at the observer. The polarization of the radiation at the observer is defined by

$$
p=\frac{P_{\|}-P_{\perp}}{P_{\|}+P_{\perp}} .
$$

In figure 1 the computations were carried out for

$$
\rho(\Delta r)=\exp \left(-\Delta r^{2} / 2 L^{2}\right),
$$

and the parameter $S_{0}$ of the diagram is given by

$$
S_{0}=h_{0} / L \text {. }
$$

In figure 2 similar computations were carried out for

$$
\rho(\Delta r)=\exp (-\Delta r / l),
$$




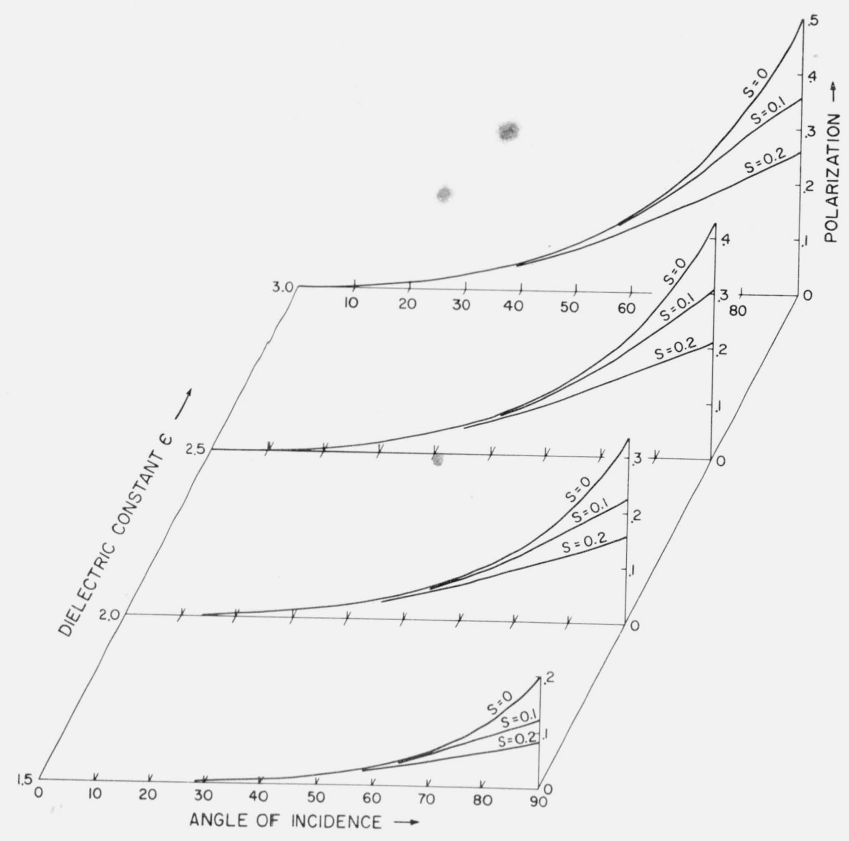

Figure 1. Polarization of thermal emission. Gaussian autocorrelation function.

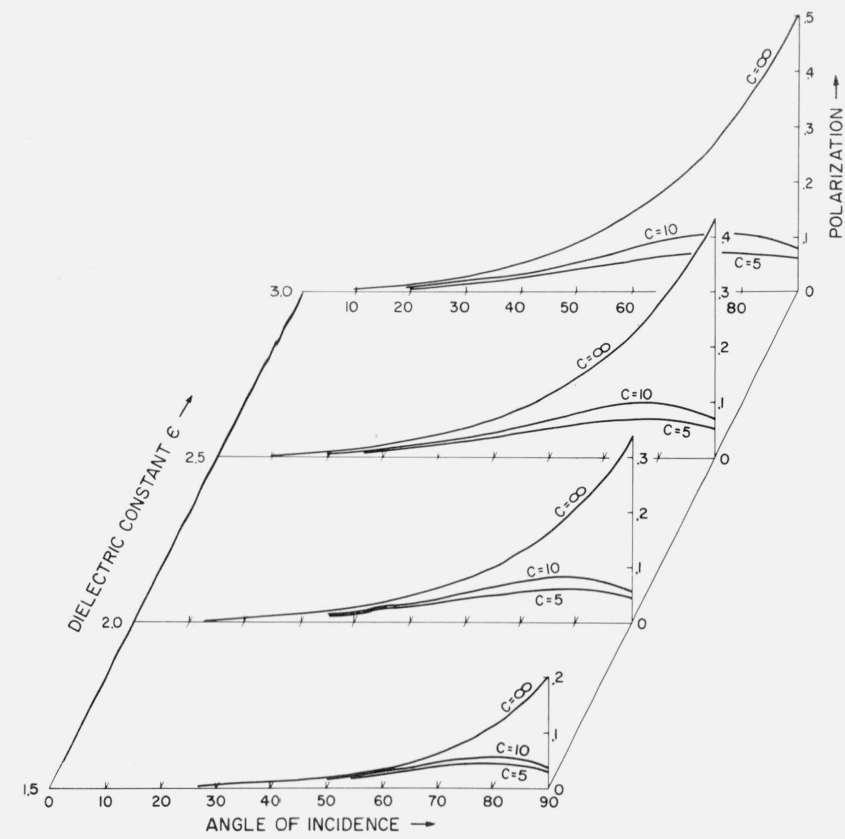

Figure 2. Polarization of thermal emission. Exponential autocorrelation function.

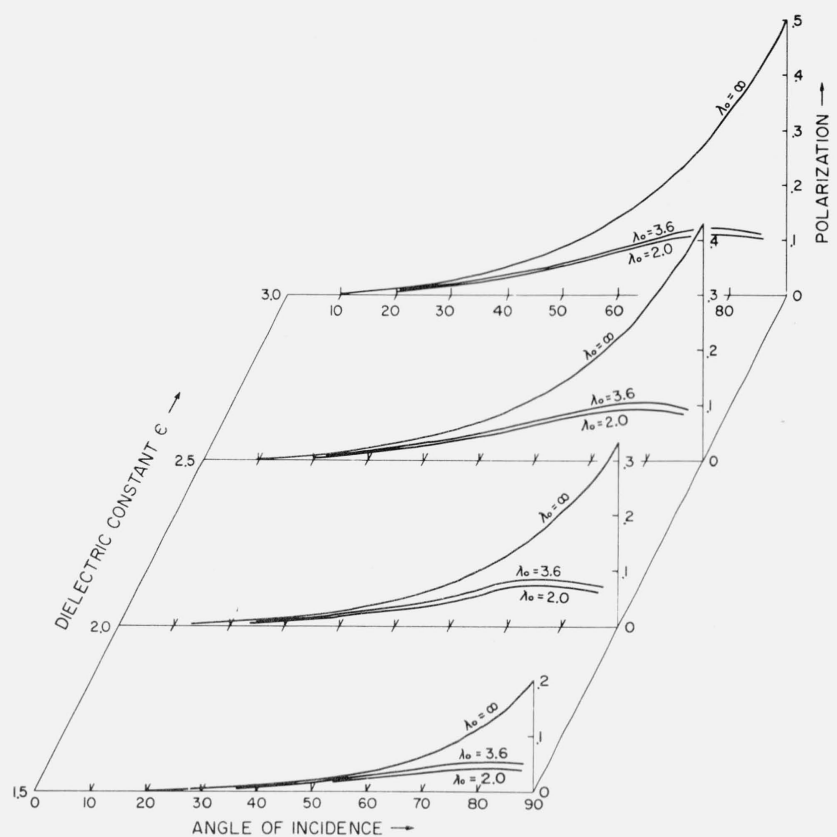

Figure 3. Polarization of thermal emission. Surface roughness as derived from lunar radar observations.

and the parameter $C$ of the diagram is defined by

$$
C^{2}=\frac{l^{2}}{h_{0}^{4} k^{2}(\sqrt{\epsilon}-1)} .
$$

Finally, the formulae were somewhat modified so that the distribution of surface slopes rather than the autocorrelation function $\rho(\Delta r)$ appeared explicitly. A distribution of surface slopes was obtained by appropriate interpolation of power-versus-range curves obtained in lunar radar studies [Evans and Pettengill, loc. cit.]. With this distribution the polarization was again computed as a function of angle of incidence to the mean surface. The results are displayed in figure 3 for $\lambda=2.0$ and $3.6 \mathrm{~cm}$.

In every case there is a pronounced effect of the roughness on the polarization, particularly when the distribution of surface slopes is wide, as in figures 2 and 3. Note in particular the slight decrease in polarization near grazing incidence for the wide angular distributions of surface slopes. This might provide an explanation for a similar effect actually observed by Mezger (this meeting) for lunar emission at $2 \mathrm{~cm}$.

Preliminary results obtained at $3.7 \mathrm{~cm}$ with the MIT Haystack antenna observing the polarization of the thermal emission from the moon indicate that the above theory goes some way toward reconciling the radar and radiometric determinations of dielectric constant, but it does not appear as if complete agreement can be reached even with this model.

(Paper 69D12-607) 